\title{
An Improved Integral Method for Prediction of Distorted Inlet Flow Propagation in Axial Compressor
}

\author{
Eddie Yin-Kwee Ng \\ School of Mechanical \& Aerospace Engineering, College of Engineering, Nanyang Technological University, 50 Nanyang Avenue, \\ Singapore 639798 \\ Email: mykng@ntu.edu.sg \\ Ningyu Liu \\ School of Mechanical \& Aerospace Engineering, College of Engineering, Nanyang Technological University, 50 Nanyang Avenue, \\ Singapore 639798 \\ Email: mpeln@nus.edu.sg
}

\section{Hong Ngiap Lim}

Propulsion Branch, Air Logistic Department, HQ RSAF, Mindef Building, Gombak Drive, Singapore 669638

Email:lhongngi@starnet.gov.sg

\section{Daniel Tan}

Propulsion Branch, Air Logistic Department, HQ RSAF, Mindef Building, Gombak Drive, Singapore 669638 Email: thuatyon@starnet.gov.sg

\section{Received 10 December 2003}

\begin{abstract}
An improved integral method is proposed and developed for the quantitative prediction of distorted inlet flow propagation through axial compressor. The novel integral method is formulated using more appropriate and practical airfoil characteristics, with less assumptions needed for derivation. The results indicate that the original integral method (Kim et al., 1996) underestimated the propagation of inlet flow distortion. The effects of inlet flow parameters on the propagation of inlet distortions as well as on the compressor performance and characteristic are simulated and analyzed. From the viewpoint of compressor efficiency, the propagation of inlet flow distortion is further described using a compressor critical performance and its associated critical characteristic. The results present a realistic physical insight to an axial-flow compressor behavior with a propagation of inlet distortion.
\end{abstract}

Keywords and phrases: improved integral method, inlet distortion propagation, axial-flow compressor, airfoil characteristics.

\section{INTRODUCTION}

Compression system is an important component of the gas turbine engine, and its performance strongly influences the performance of all other components. Distorted inlet flow in axial compressor may cause the component mismatch and instability problems, such as rotating stall or even surge, or a combination of them, which might result in catastrophic damage to the entire engine. To understand and

This is an open access article distributed under the Creative Commons Attribution License, which permits unrestricted use, distribution, and reproduction in any medium, provided the original work is properly cited. avoid compressor instability due to flow distortion, the inlet distortion and its propagation effects have received much attention over the years.

The early work about analytical and experimental descriptions of propagating stall can be traced back to midfifties. In this period, one of remarkable work was done by Marble [9]. Marble proposed a simple model to yield essential features of stall propagation, such as dependence on the extent of stalled region upon operating conditions, the pressure loss associated with stall, and the angular velocity of stall propagation. In the work done by Emmons et al. [3], an experimental investigation was performed to verify their theory of instability about the phenomena in surge and 


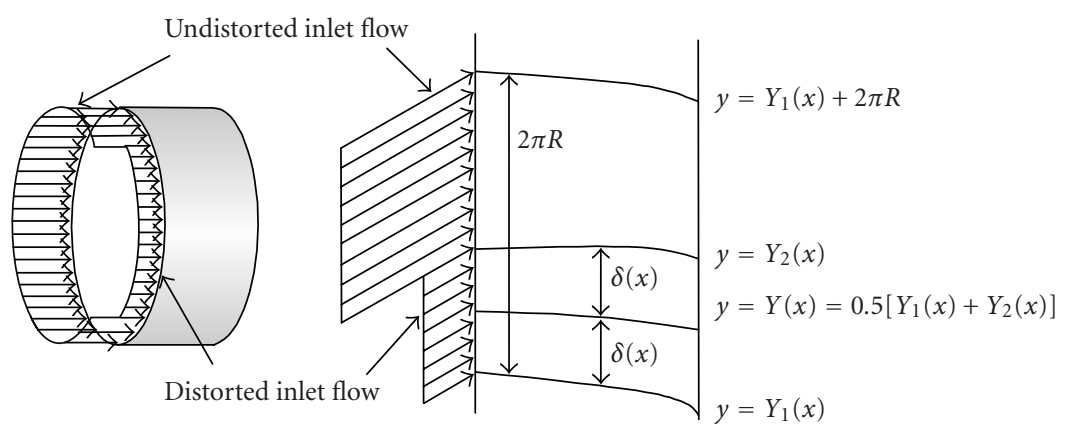

FIgure 1: The distorted inlet flow and its two-dimensional schematic used for integral method.

stall propagation. More work had been done in the recent years. Cumpsty and Greitzer [2] proposed a simple model for compressor stall cell propagation. Jonnavithula et al. [5] presented a numerical and experimental study of stall propagation in axial compressors. Longley and Hynes [8] described stability of flow through multistage axial compressors, and so on.

As to the work focusing on the analyses of distorted inlet flow, Reid [14] presented an insight into the mechanism of the compressor's response and tolerance to distortion. One of the methods used in the analyses was a linearized approach (see Plourde and Stenning [13]; Greitzer [4]), which provides quantitative information about the performance of the compressor in a circumferentially nonuniform flow. Several models (see Mikolajczak and Pfeffer [11]; Greitzer [4]), such as the parallel compressor model and its extensions (see Mazzawy [10]), were used to assess the compressor stability with inlet distortion. Stenning [16] also presented some simpler techniques for analyzing the effects of circumferential inlet distortion.

The numerical simulation of complex flows within multiple stages of turbomachinery is becoming more effective and is useful for design application today by using the advanced computers. However, a large-scale simulation with CFD codes still requires huge computing resources far exceeding the practical limits of most single-processor supercomputers. Many CFD codes have to be performed on a parallel supercomputer (see Wellborn and Delaney [18], Chen and Briley [1]). In order to rapidly predict the distorted performance and distortion attenuation of an axial compressor without using comprehensive CFD codes and parallel supercomputer, it is necessary to make some simplifications, and some elegance and detail of flow physics must be sacrificed. Kim et al. [6] successfully calculated the qualitative trend of distorted performance and distortion attenuation of an axial compressor by using an overly simplified integral method. Instead of solving a detailed flow-field problem, the integral method renders the multistage analysis as a nature part, and permits large velocity variations, including backflow. $\mathrm{Ng}$ et al. [12] developed the integral method and proposed a distortion critical line. The integral method provided a useful physical insight about the performance of the axial compressor with an inlet flow distortion. It is thus meaningful to further develop and refine this method.
In the present study, the authors further improve and develop the integral method from the previous one (Kim et al. [6]) by adopting more appropriate and realistic airfoil characteristics. The calculated results indicate that the previous integral method underestimated greatly the inlet distortion propagation. By using the newly developed integral method, an investigation is proceeded to present the effects of inlet parameters upon the downstream flow features with inlet distortion, including the inlet distortion propagation, the compressor critical performance and critical characteristic. The airfoil characteristics are also derived and discussed.

\section{THEORETICAL FORMULATION}

Consider a two-dimensional inviscid flow through an axial compressor as shown in Figure 1. In the $x, y$ plane, the circumferential extent of compressor is denoted by $y$-direction and its period is $2 \pi R$. The flowfield is divided as two parts, one is distorted flow and another is the undistorted flow. The distorted flow moves along a line $y=Y(x)$ and extends a distance $\delta(x)$ on each side of this line to form a stream tube. Thus, the distorted region in $y$-direction is from $y=Y(x)-\delta(x)$ to $y=Y(x)+\delta(x)$, and the undistorted region is from $y=Y(x)+\delta(x)$ to $y=2 \pi R+Y(x)-\delta(x)$.

The flow that is subjected to a force field can be described by the equations of continuity and motion:

$$
\begin{gathered}
\frac{\partial u}{\partial x}+\frac{\partial v}{\partial y}=0 \\
\frac{\partial\left(u^{2}\right)}{\partial x}+\frac{\partial(u v)}{\partial y}+\frac{\partial}{\partial x}\left(\frac{p}{\rho}\right)=F_{x} \\
\frac{\partial(u v)}{\partial x}+\frac{\partial\left(v^{2}\right)}{\partial y}+\frac{\partial}{\partial y}\left(\frac{p}{\rho}\right)=F_{y} .
\end{gathered}
$$

Here, $F_{x}$ and $F_{y}$ are the force in $x$ - and $y$-direction in the distorted inlet region, respectively.

For simplifying the derivation of integral equations, the coordinates system is transformed in the circumferential direction from $(x, y)$ to $(x, \eta)$ using $\eta=(y-Y(x)) / \delta(x)$. The computational domain is thus transformed into a parallel channel, as shown in Figure 2. This implies that we are considering the flow through a circular limited gap with $\delta / R \ll 1$ so that one may introduce a plane geometry. 


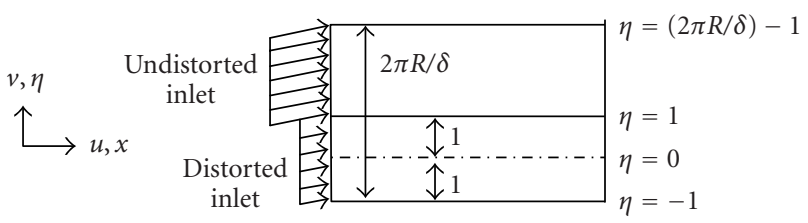

FIGURE 2: A schematic of coordinates transformation on computational domain.

The modeling technique is to integrate (1), with respect to $y$, and to determine the development of the flow from the inlet toward the downstream.

Before integrating the equations of motion and continuity in the distorted region, undistorted region and overall region, respectively, the matching of velocity profiles and pressure field should be chosen.

To illustrate the procedure of integral method, we consider a simple example.

The velocities in each of the regions are taken to be independent of $y$; whereas, the velocity components in each of the regions are defined as

$$
\begin{aligned}
u & =\alpha(x) U_{0}, \\
v & =\beta(x) V_{0}, \\
u_{0} & =\alpha_{0}(x) U_{0}, \\
v_{0} & =\beta_{0}(x) V_{0} .
\end{aligned}
$$
and

The inlet velocity has an incidence angle at inlet of $\theta_{0}$,

$$
\gamma=\tan \theta_{0}=\frac{V_{0}}{U_{0}},
$$

where $U_{0}$ and $V_{0}$ are the $x$ - and $y$-components of reference inlet velocity, respectively. The distorted velocity coefficients $\alpha(x)$ and $\beta(x)$ are the velocity fractions of the referenced inlet velocity in the distorted inlet region, and the undistorted velocity coefficients $\alpha_{0}(x)$ and $\beta_{0}(x)$ are the velocity fractions of the referenced inlet velocity in the undistorted inlet region, respectively. $u$ and $v$ are the nondimensional $x$ - and $y$ components of distorted velocity, while $u_{0}$ and $v_{0}$ are the $x$ and $y$-components of undistorted velocity, respectively.

The static pressure $p$ is taken circumferentially (vertically) uniform:

$$
\frac{p}{\rho} \equiv \frac{p}{\rho}(x)
$$

This assumption is taken in simplifying the process of derivation, which means that fluid pressure in the distorted region is fixed along $y$-direction, and this assumption would cause an overestimation of distortion level and propagation. In other words, if the decrease of static pressure is neglected, the inlet-distorted velocity that is calculated from the measured-inlet $\mathrm{v}$ total pressure will be smaller than the reality. Therefore, the difference of the velocities between two regions at inlet will be increased compared with the reality, and the inlet distortion would be overestimated. The predicted results of propagation of distortion would be larger than the real one, and hence provide a wider safety margin. The more the compressor's stage number (longer axial scale) is, the higher the magnitude of overestimated distortion would be.

In the equations of motion and continuity, the force components $F_{x}$ and $F_{y}$ are the replacements of the equivalent terms acting on the blades as shown in Figure 3. The multistages therefore become a natural part of compressor. The following are the definitions of the forces acting on the stator and rotor in the region of distortion according to the blade element theory (replacing $u$ and $v$ by $u_{0}$ and $v_{0}$ in the undistorted region):

$$
\begin{gathered}
F_{\perp}^{s}=\frac{C_{l}}{2}\left(u^{2}+v^{2}\right), \\
F_{/ /}^{s}=\frac{C_{d}}{2}\left(u^{2}+v^{2}\right), \\
F_{\perp}^{r}=\frac{C_{l}}{2}\left[u^{2}+(\omega R-v)^{2}\right], \\
F_{/ /}^{r}=\frac{C_{d}}{2}\left[u^{2}+(\omega R-v)^{2}\right] .
\end{gathered}
$$

Here, $F_{\perp}$ and $F_{/ /}$are parallel and normal force components, respectively; $\omega$ is rotor angle velocity; $C_{l}$ and $C_{d}$ are the lift and drag coefficients, respectively. The force components for a unit circumferential distance of a complete stage are

$$
\begin{gathered}
F_{x}=\frac{\lambda_{r}}{\bar{\lambda}}\left(F_{\perp}^{r} \sin \theta_{r}-F_{/ /}^{r} \cos \theta_{r}\right)+\frac{\lambda_{s}}{\bar{\lambda}}\left(F_{\perp}^{s} \sin \theta_{s}-F_{/ /}^{s} \cos \theta_{s}\right), \\
F_{y}=\frac{\lambda_{r}}{\bar{\lambda}}\left(F_{\perp}^{r} \cos \theta_{r}+F_{/ /}^{r} \sin \theta_{r}\right)+\frac{\lambda_{s}}{\bar{\lambda}}\left(-F_{\perp}^{s} \cos \theta_{s}-F_{/ /}^{s} \sin \theta_{s}\right),
\end{gathered}
$$

where the superscript and subscript $s$ denote the stator; the superscript and subscript $r$ denote the rotor; $\lambda_{r} / \bar{\lambda}$ and $\lambda_{s} / \bar{\lambda}$ are the relative length of rotor and stator in a single stage, respectively. Here, we assume that $\bar{\lambda}=\lambda_{r}+\lambda_{s}$. The angles $\theta_{s}$ and $\theta_{r}$ are the local incidence angles with respect to stator and rotor. For example, in distorted region,

$$
\begin{gathered}
\tan \theta_{s}=\frac{v}{u}, \\
\tan \theta_{r}=\frac{(\omega R-v)}{u} .
\end{gathered}
$$




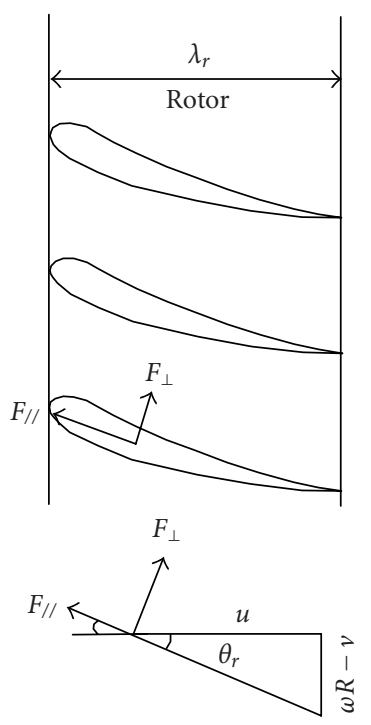

(a)
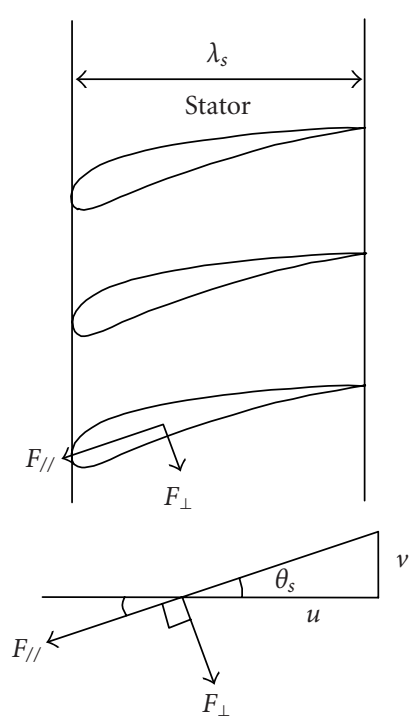

(b)

FIGURE 3: The force diagram and velocities in the compressor stage.

The $(u, v)$ will be replaced by $\left(u_{0}, v_{0}\right)$ in undistorted region.

By substituting of (2), (3), (5), and (7) into (6), and simplifying the resulting equations by using $\sigma=\omega R / V_{0}$, we obtain

$$
\begin{aligned}
\frac{F_{x}}{U_{0}^{2}}= & \frac{\lambda_{r}}{2 \bar{\lambda} \sigma^{2} \gamma^{2}}\left[C_{l}(\sigma-\beta) \gamma-C_{d} \alpha\right] \sqrt{\alpha^{2}+(\sigma-\beta)^{2} \gamma^{2}} \\
& +\frac{\lambda_{s}}{2 \bar{\lambda} \sigma^{2} \gamma^{2}}\left(C_{l} \beta \gamma-C_{d} \alpha\right) \sqrt{\alpha^{2}+\beta^{2} \gamma^{2}}, \\
\frac{F_{y}}{U_{0}^{2}}= & \frac{\lambda_{r}}{2 \bar{\lambda} \sigma^{2} \gamma^{2}}\left[C_{l} \alpha+C_{d}(\sigma-\beta) \gamma\right] \sqrt{\alpha^{2}+(\sigma-\beta)^{2} \gamma^{2}} \\
& -\frac{\lambda_{s}}{2 \bar{\lambda} \sigma^{2} \gamma^{2}}\left(C_{l} \alpha+C_{d} \beta \gamma\right) \sqrt{\alpha^{2}+\beta^{2} \gamma^{2}} .
\end{aligned}
$$

Similarly, the force components in the undistorted region $F_{x, 0}$ and $F_{y, 0}$ can be obtained using expression of undistorted velocity coefficients $\alpha_{0}$ and $\beta_{0}$ in $x$-direction and $y$-direction, respectively. Here, the equations for the force components are different from the previous expressions (see Kim et al. [6]). The current effort is derived using an exact blade element theory. On the other hand, unlike the oversimplified procedure with the constants lift and drag coefficients, the more practical coefficients are to be applied according to the experimental results of airfoil sections (see Abbott and Von Doenhoff [17]).

In the distorted region, because the boundaries are streamlines, we obtain the following equations by integrating
(1) along $\eta$-direction:

$$
\begin{gathered}
\delta \int_{-1}^{1} u d \eta=\text { constant, } \\
\frac{d}{d x}\left[\int_{-1}^{1} u^{2} \delta d \eta\right]+\int_{-1}^{1}\left\{\delta \frac{d}{d x}-\left(Y^{\prime}+\delta^{\prime} \eta\right) \frac{\partial}{\partial \eta}\right\} \frac{p}{\rho} d \eta \\
=\int_{-1}^{1} F_{x} \delta d \eta \\
\frac{d}{d x}\left[\int_{-1}^{1} u v \delta d \eta+\frac{p}{\rho}(x, 1)-\frac{p}{\rho}(x,-1)\right]=\int_{-1}^{1} F_{y} \delta d \eta .
\end{gathered}
$$

From (9), the product of $\delta \alpha$ is a constant. For ease in derivation, we take

$$
\frac{\delta \alpha}{\pi R} \equiv K_{1}
$$

From (10) and (11), we obtain

$$
\begin{gathered}
\alpha \frac{d \alpha}{d x}+\frac{1}{U_{0}^{2}} \frac{d}{d x}\left(\frac{p}{\rho}\right)=\frac{F_{x}}{U_{0}^{2}}, \\
\alpha \frac{d \beta}{d x}=\frac{1}{\gamma}\left(\frac{F_{y}}{U_{0}^{2}}\right) .
\end{gathered}
$$

In the undistorted region, by integrating the equations of motion in $[1,(2 \pi R / \delta)-1]$, we thus obtain

$$
\begin{gathered}
\alpha_{0} \frac{d \alpha_{0}}{d x}+\frac{1}{U_{0}^{2}} \frac{d}{d x}\left(\frac{p}{\rho}\right)=\frac{F_{x, 0}}{U_{0}^{2}}, \\
\alpha_{0} \frac{d \beta_{0}}{d x}=\frac{1}{\gamma}\left(\frac{F_{y, 0}}{U_{0}^{2}}\right) .
\end{gathered}
$$


Next, with integration of the continuity equation over the whole region,

$$
\delta \int_{-1}^{1} \alpha U_{0} d \eta+\delta \int_{1}^{(2 \pi R / \delta)-1} \alpha_{0} U_{0} d \eta \equiv \text { constant }
$$

yields

$$
\frac{\left[2 \delta \alpha U_{0}+(2 \pi R-2 \delta)\left(\alpha_{0} U_{0}\right)\right]}{\left(2 \pi R U_{0}\right)} \equiv K_{0} .
$$

Using (12),

$$
K_{1}+\left(\frac{1-K_{1}}{\alpha}\right) \alpha_{0}=K_{0},
$$

rearranged as

$$
\alpha_{0}=\frac{\alpha\left(K_{0}-K_{1}\right)}{\alpha-K_{1}} .
$$

Differentiating the above equation will result in

$$
\frac{d \alpha_{0}}{d x}=K_{2} \frac{d \alpha}{d x}
$$

where

$$
K_{2}(\alpha)=\frac{K_{1}\left(K_{1}-K_{0}\right)}{\left(\alpha-K_{1}\right)^{2}} .
$$

The integrated result of equation of motion in the overall region is equal to the combined results in both distorted and undistorted regions. In $x$-direction, by combining (13) and (15), we obtain

$$
\alpha \frac{d \alpha}{d x}-\alpha_{0} \frac{d \alpha_{0}}{d x}=\frac{F_{x}-F_{x, 0}}{U_{0}^{2}} .
$$

Substituting (20) and (21) into (23) yields

$$
\alpha \frac{d \alpha}{d x}=\frac{1}{K_{3}} \frac{F_{x}-F_{x, 0}}{U_{0}^{2}},
$$

where

$$
K_{3}(\alpha)=1+\frac{K_{2}\left(K_{1}-K_{0}\right)}{\alpha-K_{1}} .
$$

From the above results, we can rearrange the five ordinary differential equations (13), (14), (16), (21), and (24) as follows:

$$
\begin{gathered}
\alpha \frac{d \alpha}{d x}=\frac{1}{K_{3}}\left(\frac{F_{x}-F_{x, 0}}{U_{0}^{2}}\right), \\
\alpha \frac{d \beta}{d x}=\frac{1}{\gamma}\left(\frac{F_{y}}{U_{0}^{2}}\right),
\end{gathered}
$$

$$
\begin{gathered}
\frac{d \alpha_{0}}{d x}=K_{2} \frac{d \alpha}{d x} \\
\alpha_{0} \frac{d \beta_{0}}{d x}=\frac{1}{\gamma}\left(\frac{F_{y, 0}}{U_{0}^{2}}\right), \\
\frac{d}{d x}\left(\frac{p}{\rho}\right)=U_{0}^{2}\left(\frac{F_{x}}{U_{0}^{2}}-\alpha \frac{d \alpha}{d x}\right) .
\end{gathered}
$$

The above-improved ordinary differential equations include five variables. They are two distorted velocity coefficients $\alpha(x)$ and $\beta(x)$, two undistorted velocity coefficients $\alpha_{0}(x)$ and $\beta_{0}(x)$, and one static pressure $(p / \rho)$. These integral equations can describe the development of both distorted and undistorted regions, as well as (and most important) the progression of pressure in the compressor. The relative magnitude of vertical extension of distorted region is

$$
\xi(x)=\frac{\delta(x)}{\pi R}=\frac{K_{1}}{\alpha(x)} .
$$

With calculation of the four velocity coefficients in solving the integral equations, the size of distorted region $\xi(x)$ can then be computed using (31).

There are two significant improvements between the current integral equation and the previous attempt. One of them is the force expression. In the previous papers, for simplicity, the lift and drag coefficients were assumed as constants and then an artificial term including the angle of attack was inserted to correct the error induced by the assumption. This simplification resulted in the vanishing of lift and drag forces simultaneously at some incidence angles, which is in a real flow unrealistic (nonphysical). We thus employ actual airfoil characteristics in expressing the force components without any significant assumption, which should produce flow with better physics.

The second contribution is in the derivation of integral equation. Kim et al. [6] used an equation with conservative form in distorted region but nonconservative form in undistorted region. We derive the equations in strong conservative form for both regions. This permits different results with the changes of integral equation (29), the inclusion of variation in pressure-density ratio (30), and the associated parametric expression (25). These equations are different from the previous effort.

\section{RESULTS AND DISCUSSION}

The original partial differential equation (1) has been coded and solved (see Liu et al. [7]), and the results were compared with that of Kim et al. (see [6]). Based on these research works, the current improved strong conservative form of integral equation for both distorted and undistorted regions with force expression (8) are then solved by using fourthorder Runge-Kutta method with the following initial conditions: $\lambda_{r}=\lambda_{s}=0.5 \lambda, \alpha_{0}(0)=\beta_{0}(0)=1.0$, and $\alpha(0)=\beta(0)$. From the experimental test cases (see Serovy and Dring [15]), the rotor blade speed is $\omega R=36.6 \mathrm{~m} / \mathrm{s}, \sigma=2$, and the airfoil blade section is NACA 65-series. 


\subsection{Lift and drag coefficients}

The most significant part in the current effort is the development of the application with airfoil characteristics.

In the first step, we release/avoid the assumption of constant lift and drag coefficients, as well as the need of correcting terms in force components. Then we collate the necessary experimental data of both lift and drag coefficients. Finally, by curve fitting procedure, two sets of data are summarized as two cure formulas suitable to be employed as the expression of force components.

The experimental data of NACA 65-series airfoil (see Abbott and Von Doenhoff [17]) indicates that the lift coefficient has a linear relationship with the angle of attack in a normal range $\left[-8^{\circ}, 10^{\circ}\right]$, while the drag coefficient is the second or the higher power of the lift coefficient, and there is a much smaller drag coefficient with a small angle of attack $\left[-1^{\circ}, 2^{\circ}\right]$. For example, a NACA 65-209 wing section with a lower Reynolds number $\mathrm{Re}=3.0 \times 10^{6}$, the data can be described as

$$
\begin{aligned}
C_{l}= & 0.1062 \tilde{\alpha}+0.15 \quad\left(-8^{\circ} \leq \tilde{\alpha} \leq 10^{\circ}\right), \\
C_{d}= & 0.6518 \times 10^{-2}-0.96313 \times 10^{-3} C_{l}, \\
& +0.79495 \times 10^{-2} C_{l}^{2}-0.60949 \times 10^{-2} C_{l}^{3} \\
& -0.20416 \times 10^{-3} C_{l}^{4}+0.44918 \times 10^{-2} C_{l}^{5} \\
& \left(\tilde{\alpha}<-1^{\circ}, \tilde{\alpha}>2^{\circ}\right), \\
C_{d}= & 0.0043 \quad\left(-1^{\circ} \leq \tilde{\alpha} \leq 2^{\circ}\right) .
\end{aligned}
$$

Here, $\tilde{\alpha}$ is the wing section angle of attack. In the present case, (32a) is obtained by linear fitting, and (32b) is calculated by Chebyshev curve fitting. Using (32) in (8), the force components can thus be obtained.

\subsection{Inlet-distorted velocity coefficient}

According to the previous paper (Ng et al. [12]), the inletdistorted velocity coefficient and incident angle are the essential parameters affecting the inlet-distortion propagation. Firstly, the effect of variation in inlet-distorted velocity coefficient $\alpha(0)$ is analyzed here to show what role does it play in the current novel integral method.

To facilitate in discussion of distortion quantitatively, a distortion level is defined as

$$
\Gamma(x)=1-\frac{\alpha(x)}{\alpha_{0}(x)} .
$$

The smaller $\alpha(0)$ means higher inlet distortion level. It is obvious that the definition of distortion level in representing the relative distortion is more intuitive than using the distorted velocity coefficient. For example, in the case with $\alpha_{0}(0)=1.0$ and $\alpha(0)=0.1$, the distortion levels at inlet are $\Gamma(0)=0.9$. This is a severe distortion case with a high initial distortion level. While $\alpha(0)=1.0$ will result in a $\Gamma(0)=0.0$, and hence zero distortion.

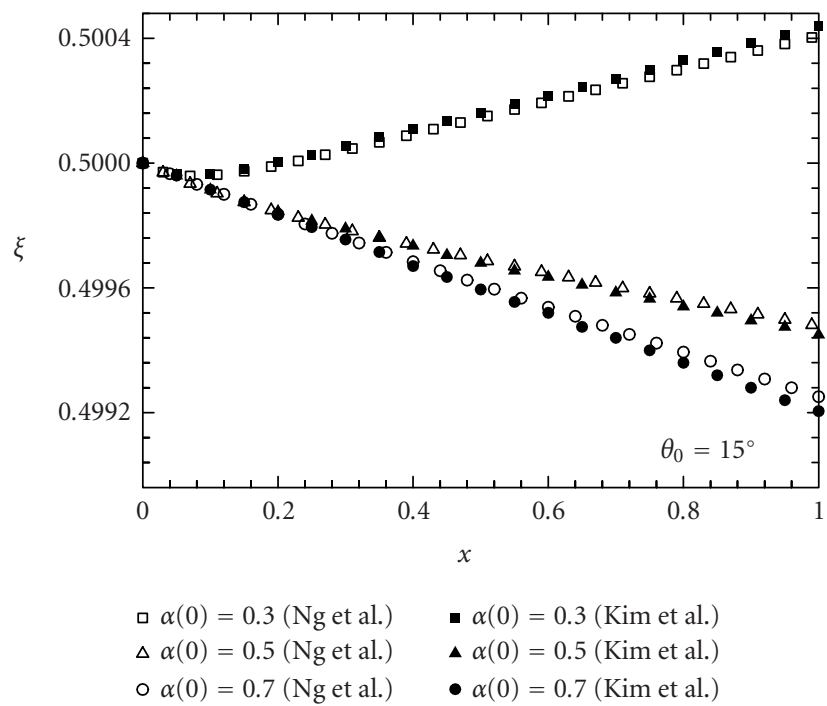

Figure 4: A comparison of distorted flow propagation between the results of $\mathrm{Ng}$ et al. [12] and those of Kim et al. [6].

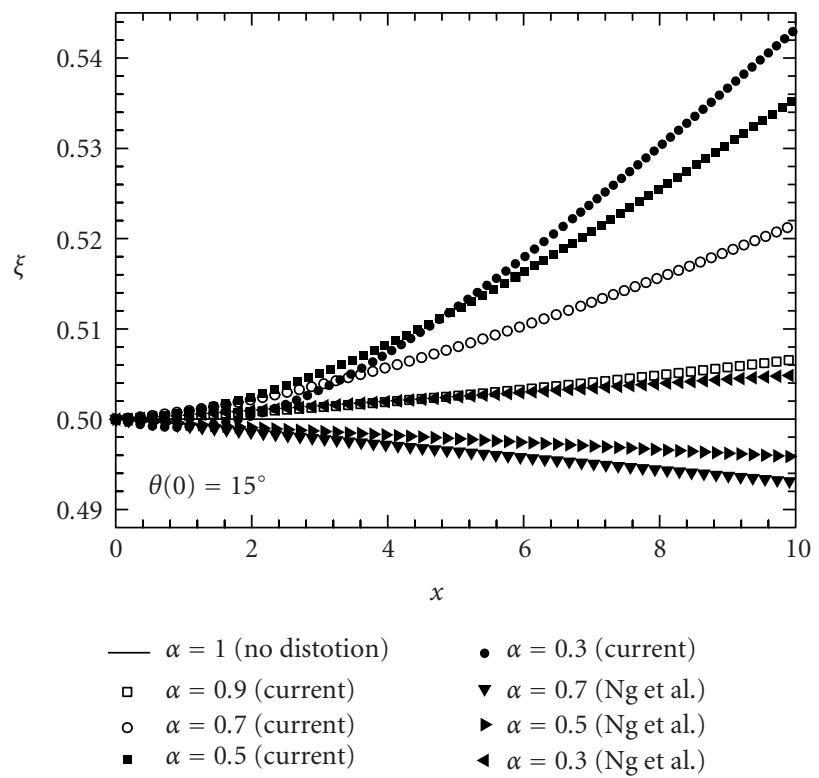

FIGURE 5: The inlet distortion propagates along axial direction with different inlet distortion levels.

Figure 4 shows that the authors' previous works (see $\mathrm{Ng}$ et al. [12]) are in good agreement with that of Kim et al. [6], which indicates that the propagation of inlet distortion with a bigger inlet distortion level will grow and vice versa. However, the results using the present novel integral method suggest a different conclusion. From Figure 5, the novel method provides a more serious propagation of inlet distortion. On the other hand, unlike the cases in Figure 4, the present results indicate that for any inlet distortion level, the size of distorted region will grow along $x$-direction. In other words, using a force with simplified assumption, the integral method would underestimate the propagation of inlet distortion. 


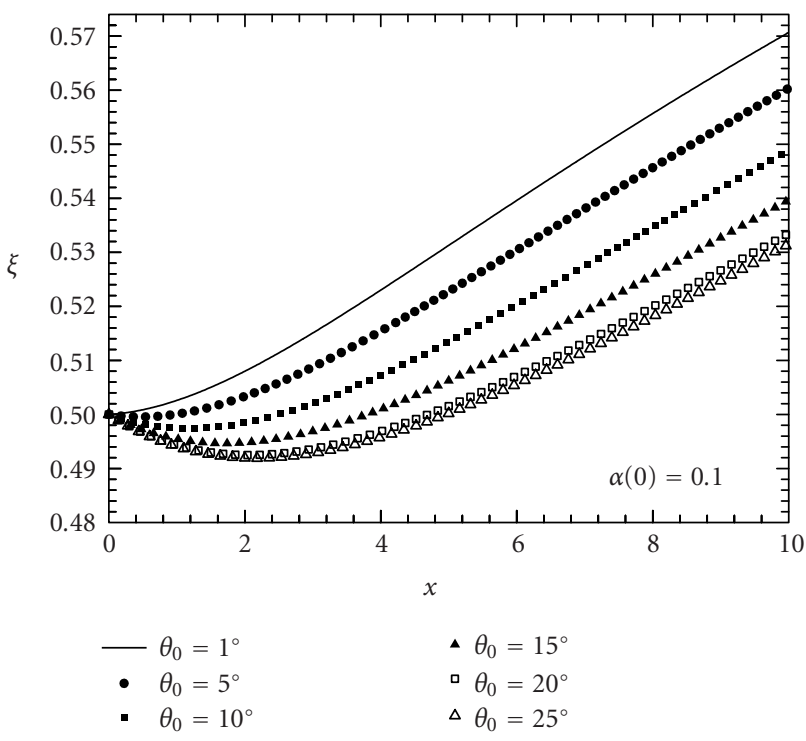

FIgURE 6: The inlet distortion propagates along axial direction at smaller inlet incident angles, $\theta_{0} \leq 25^{\circ}$.

Figure 5 indicates that a distorted region size will increase with an increasing of distortion. Higher inlet distortion level (or smaller inlet-distorted velocity coefficient) results in a more severe propagation of distortion.

\subsection{Inlet incident angle}

To study on extreme case, a higher inlet distortion level $(\Gamma(0)=0.9$, or $\alpha(0)=0.1)$ is fixed during the analysis for variation in distortion with different inlet incident angles.

The calculation shows that the inlet distortion will grow for a multistage compressor in any inlet incident angle. However, this growing magnitude is not a monotonous function of inlet incident angle only. The increment of distorted region size at outlet $\xi(x=10)$ will decrease with the increasing of inlet incident angle before about $\theta_{0}=25^{\circ}$, and then will increase with the increasing of incident angle. Therefore, the results are presented in two figures, Figures 6 and 7. In Figure 6, smaller inlet incident angle induces a larger propagation of inlet distortion. Because a small inlet incident angle induces a large vertical flow in distorted region as shown in Figure 8 , thus induces a small axial-distorted velocity coefficient from (27), and then a large size of distorted region from (31). On the contrary, when the inlet incident angle grows to a large value, $\theta_{0}=25^{\circ}-30^{\circ}$ in the current case, the increment of distorted region size at outlet will increase with the increasing of the inlet incident angle as shown in Figure 7. This is because with a larger inlet incident angle, the vertical flow in distorted region tends to decrease (Figure 9).

\subsection{Propagation of distortion level}

The inlet distortion varying along axial direction with different inlet velocity coefficients or inlet incidence angles has been investigated. However, what would be observed from the viewpoint at outlet for a ten-stage compressor with differ-

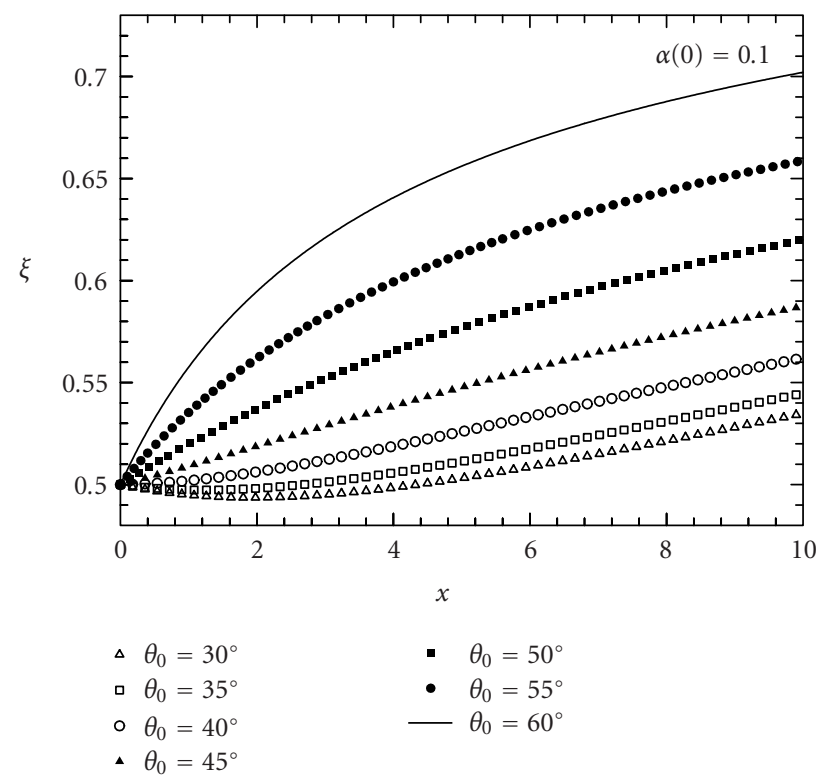

FIgURE 7: The inlet distortion propagates along axial direction at higher inlet incident angles, $\theta_{0} \geq 25^{\circ}$.

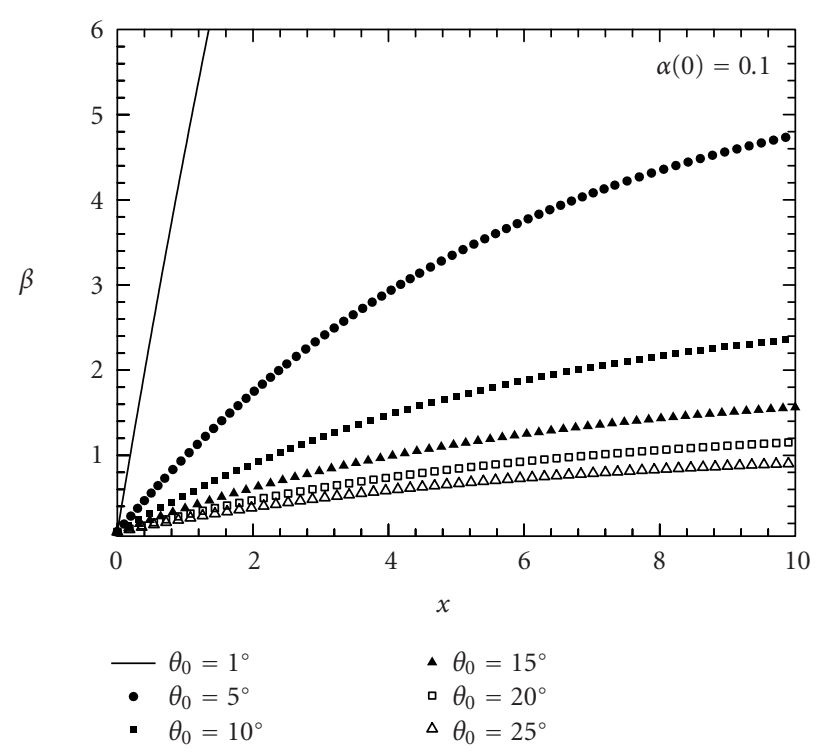

Figure 8: The vertical distorted velocity coefficient $\beta$ propagates along axial direction at smaller inlet incident angles, $\theta_{0} \leq 25^{\circ}$.

ent inlet velocity coefficients, inlet incidence angles, or inletdistorted region sizes? Figures 10 and 11 indicate that the outlet size of distorted region is larger for a case with higher inlet distortion level regardless of what the inlet size of the distorted region is. On the other hand, for a case with higher inlet distortion level, the radius of curvature of outlet size of the distorted region tends to be increased whatever the inlet size of distorted region is.

When inlet incidence angle is very small, the outlet size of distorted region will decrease with the decreasing of inlet 


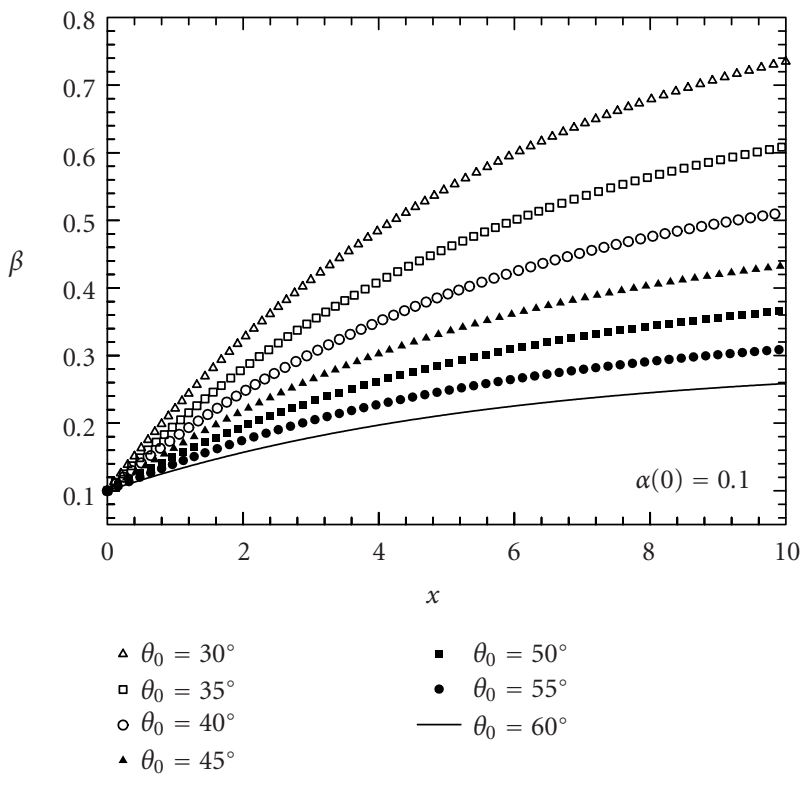

FIgURE 9: The vertical distorted velocity coefficient $\beta$ propagates along axial direction at higher inlet incident angles, $\theta_{0} \geq 25^{\circ}$.

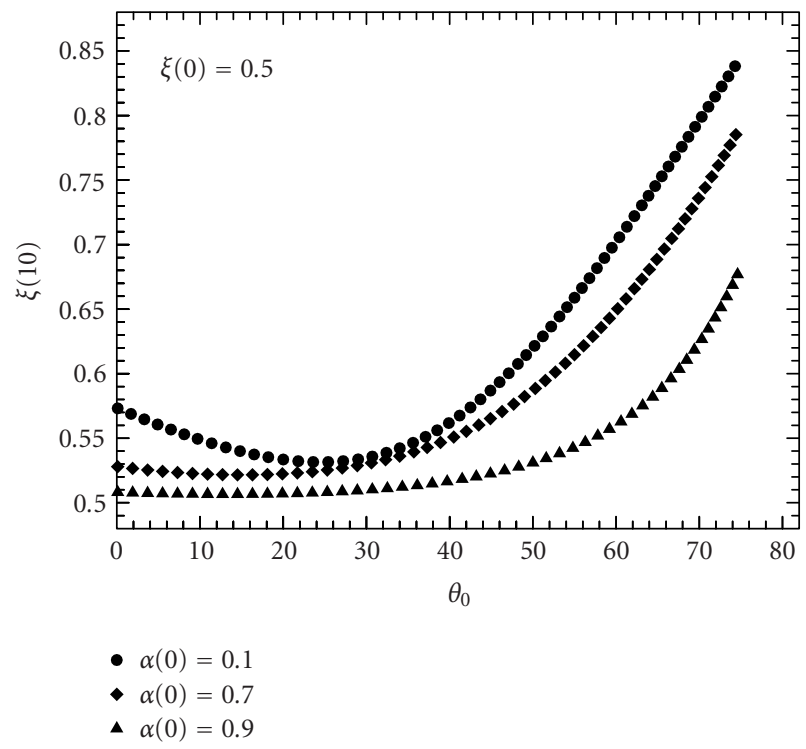

FIGURE 10: The predicted outlet size of distorted region versus $\theta_{0}$ with higher inlet size of distorted region of 0.5 .

distortion level. With the increase of inlet incidence angle, the peak point of outlet size of distorted region will move forward along $\alpha(0)$ axes (Figures 12 and 13). In other words, the peak point of $\xi(10)$ corresponds to an increased value of $\alpha(0)$ at a higher inlet incidence angle.

To ease in comparing the results between different inlet sizes of distorted region, we define the level of distortion propagation by the difference between the sizes of outlet and inlet-distorted regions such as $[\xi(10)-\xi(0)]$ in the current case. With this definition, we can arrange the results with different inlet sizes of distorted region in a single plot as shown

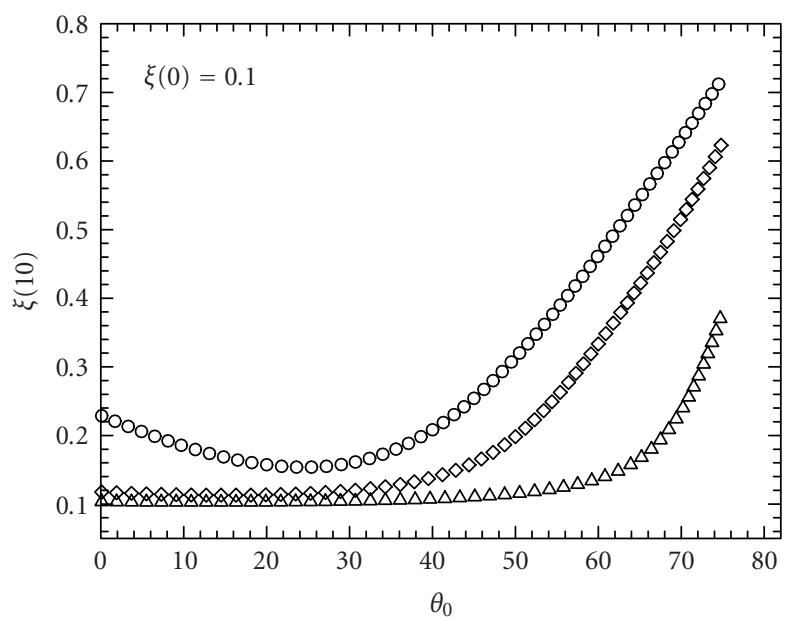

$\diamond \alpha(0)=0.1$
$\diamond \alpha(0)=0.7$
$\triangle \alpha(0)=0.9$

Figure 11: The predicted outlet size of distorted region versus $\theta_{0}$ with smaller inlet size of distorted region of 0.1 .

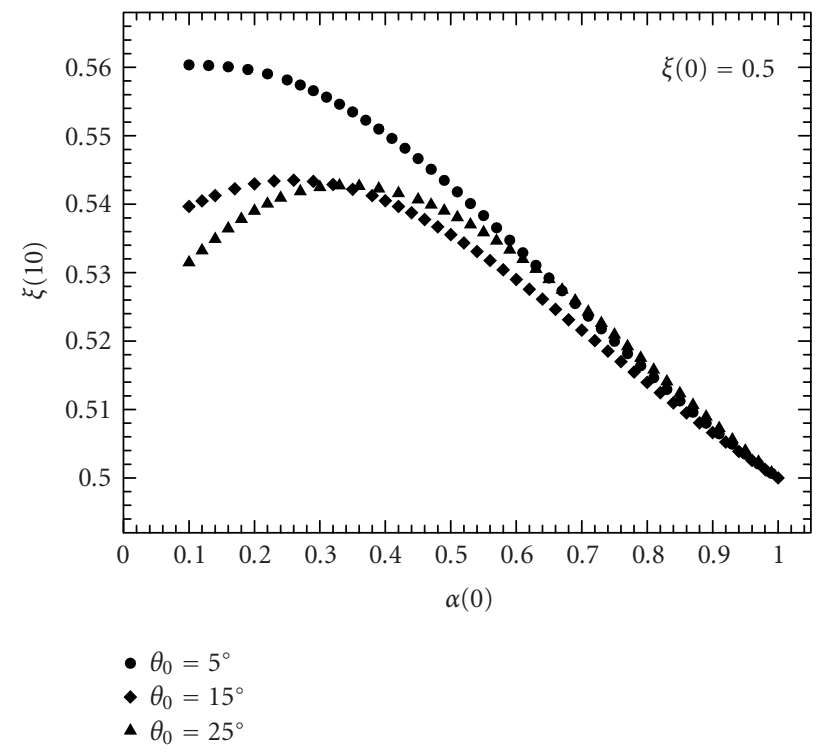

FIGURE 12: The predicted outlet size of distorted region versus $\alpha(0)$ with higher inlet size of distorted region of 0.5.

in Figures 14 and 15. Both figures illustrate that for a higher inlet incidence angle, more severe distortion propagation occurs with a larger inlet size of distorted region. On the contrary, for a lower inlet incidence angle with $\theta_{0} \leq 25^{\circ}$, a higher level of distortion propagation occurs with a smaller inlet size of distorted region $\xi(0)$.

\subsection{Compressor characteristics}

The total pressure ratio and the static pressure rise of compressor are investigated to study the effects of inlet parameters on the compressor performance and characteristics. 


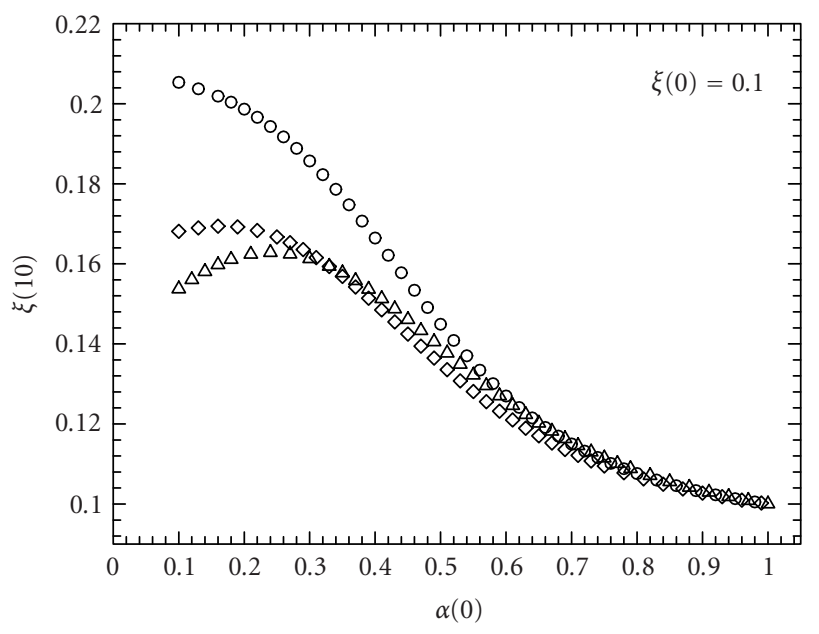

$\circ \theta_{0}=5^{\circ}$

$\diamond \theta_{0}=15^{\circ}$

$\Delta \theta_{0}=25^{\circ}$

FIGURE 13: The predicted outlet size of distorted region versus $\alpha(0)$ with smaller inlet size of distorted region of 0.1 .

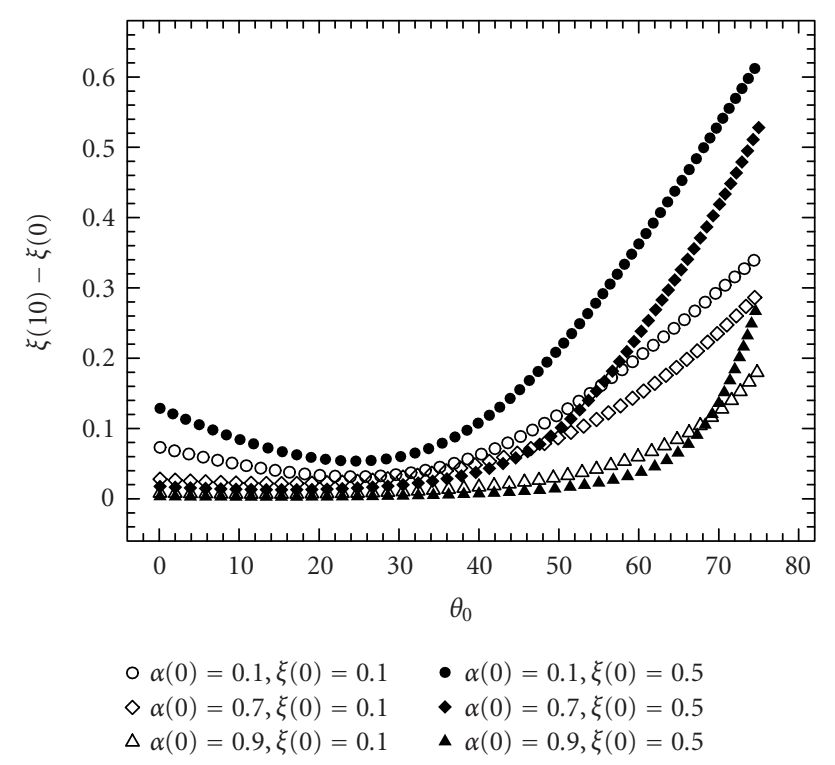

FIGURE 14: The level of distortion propagation versus inlet incidence angle.

Figure 16 indicates that a smaller inlet incidence angle causes a higher total pressure ratio, and a smaller inletdistorted velocity coefficient $\alpha(0)$ or a higher inlet distortion level $\Gamma(0)$ induces a higher total pressure ratio. However, the inlet size of distorted region has no obvious effect on the total pressure ratio.

In the small and medium inlet incidence angles, the static pressure rises are almost the same with a constant value as shown in Figure 17. The static pressure rise does not change whatever the inlet-distorted velocity coefficient $\alpha(0)$ and the inlet size of distorted region $\xi(0)$ are. However, with a larger inlet incidence angle $\left(\theta_{0}>45^{\circ}\right)$, the static pressure rises

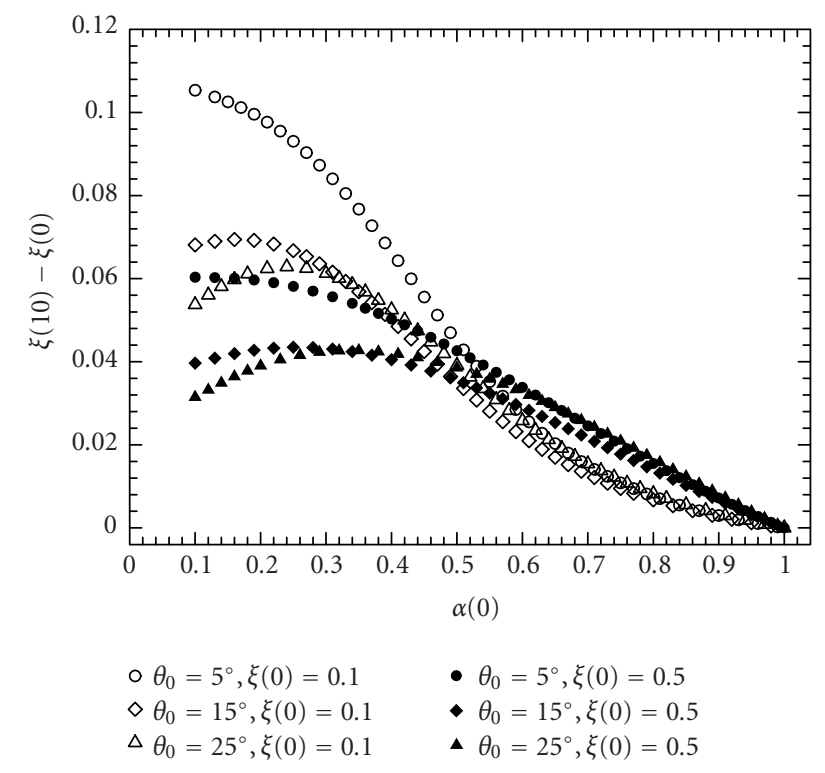

Figure 15: The level of distortion propagation versus inletdistorted velocity coefficient.

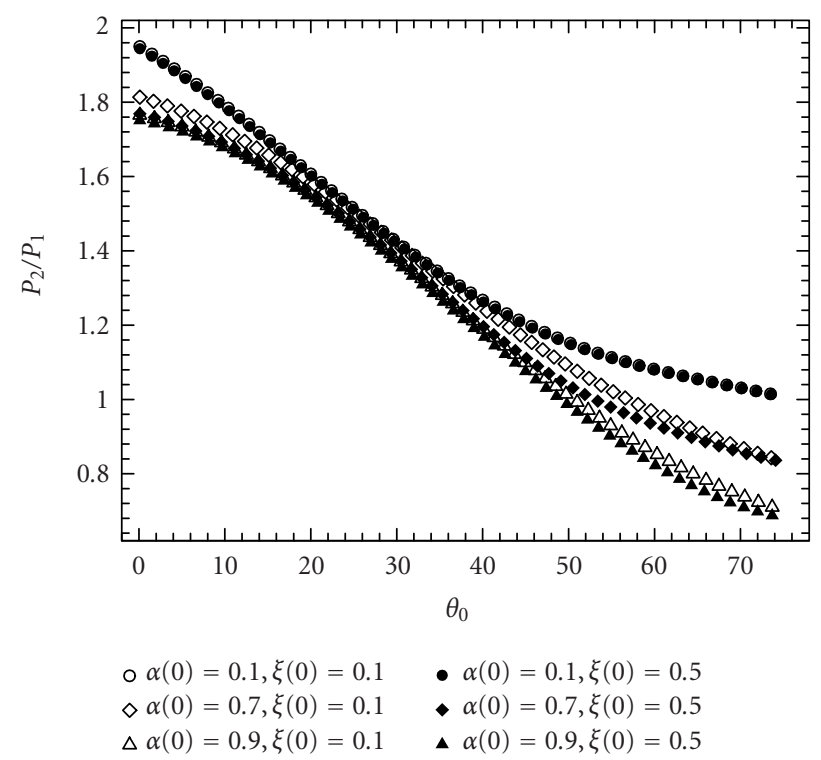

FIGURE 16: The computed compressor total pressure ratio versus inlet incidence angle.

sharply with a higher inlet-distorted velocity coefficient.

By keeping eyes on the smaller inlet incidence angles with $\theta_{0} \leq 25^{\circ}$ and changing $\alpha(0)$ from 0 to 1.0 , we can obtain the compressor characteristics as summarized in Figures 18 and 19. The larger inlet size of distorted region $\xi(0)$ produces a larger change in mass flow rate. Meanwhile, a smaller incidence angle produces a higher pressure rise. This is easy to understand because $\sigma=\omega R / V_{0}$, and we set $\sigma$ and $\omega R$ as constants, thus the vertical reference velocity $V_{0}$ is a constant too. From (3), $\tan \theta_{0}=V_{0} / U_{0}$, a smaller $\theta_{0}$ will produce a larger $U_{0}$ for a constant value of $V_{0}$, thus a higher pressure rise. 


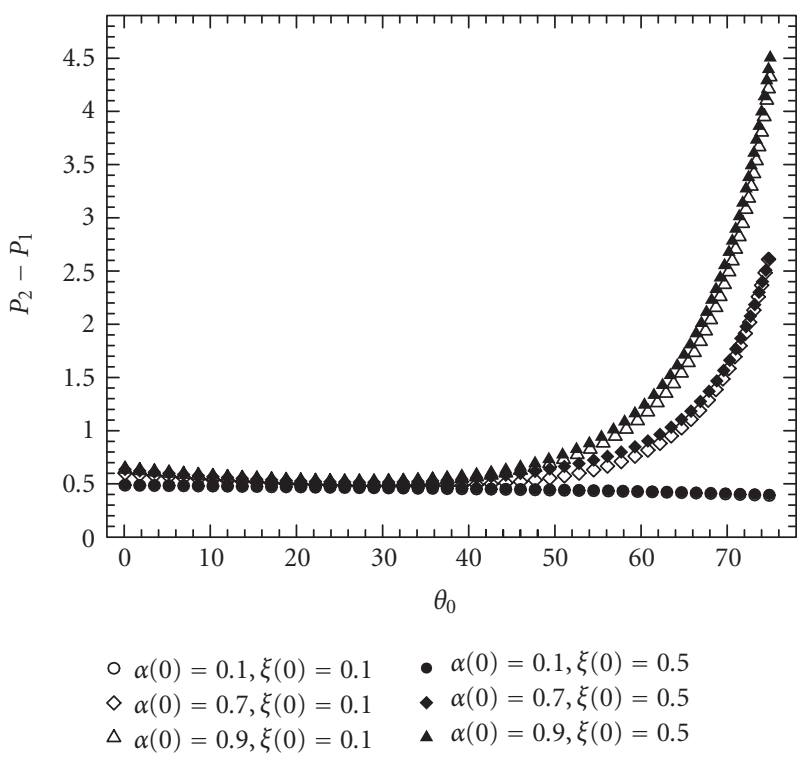

FIGURE 17: The computed compressor static pressure rise versus inlet incidence angle.

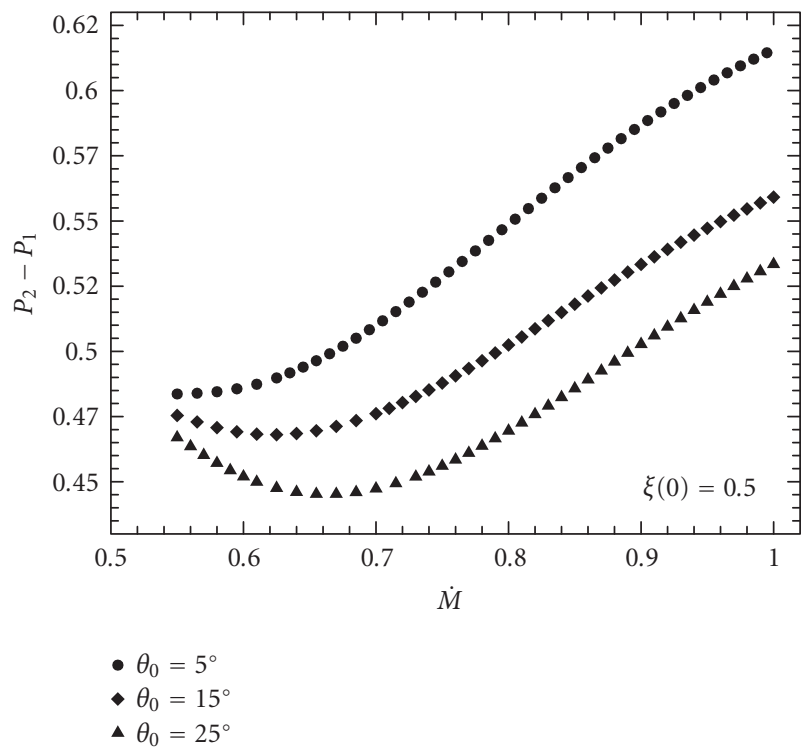

FIGURE 18: The simulated compressor characteristics at higher inlet size of distorted region of 0.5 .

\subsection{Argument for airfoil characteristics}

As mentioned in Section 3.1, we propose the airfoil lift and drag coefficients to be described as the functions of the angle of attack according to the wing section theory and experimental data:

$$
\begin{aligned}
C_{l} & =f_{1}(\tilde{\alpha}), \\
C_{d} & =f_{2}\left(C_{l}\right) .
\end{aligned}
$$

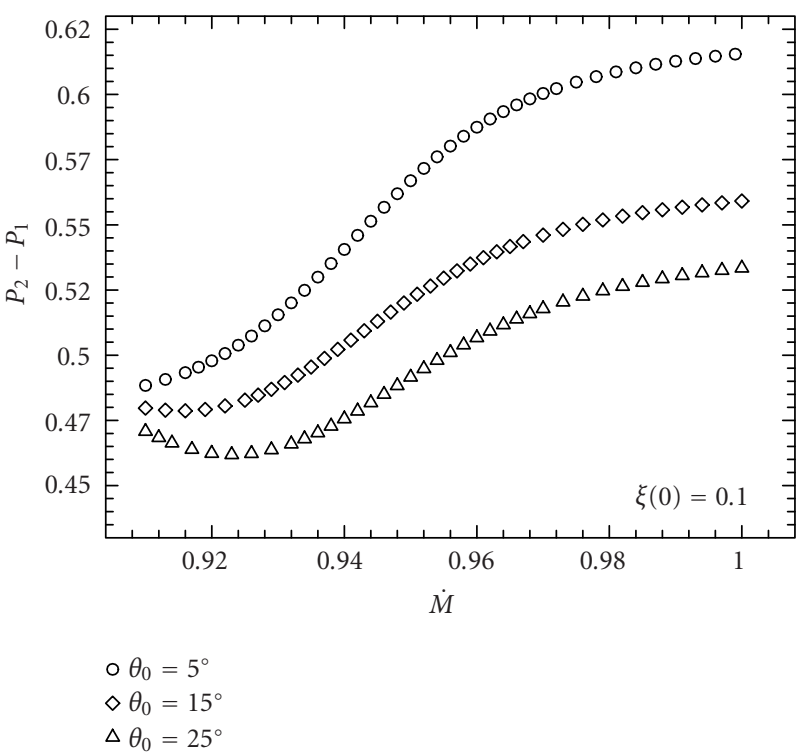

FIGURE 19: The simulated compressor characteristics at smaller inlet size of distorted region of 0.1 .

Here, the angles of attack for rotor and stator are described as follows in the distorted region (Figure 3):

$$
\begin{gathered}
\tilde{\alpha}_{r}=\tan ^{-1}\left(\frac{\sigma-\beta}{\alpha} \gamma\right), \\
\tilde{\alpha}_{s}=\tan ^{-1}\left(\frac{\beta}{\alpha} \gamma\right) .
\end{gathered}
$$

The angles of attack in the undistorted region can also be obtained with expression of undistorted velocity coefficients $\alpha_{0}$ and $\beta_{0}$.

From (26) and (31), we have

$$
\frac{d \xi}{d x}=\frac{1}{\left(K_{1}^{2} / \xi^{3}\right)+\left(K_{1}-K_{0}\right) /(1-\xi)^{3}} \cdot \frac{F_{x, 0}-F_{x}}{U_{0}^{2}} .
$$

Because the first term of the right-hand side is positive, $\left(K_{1}^{2} / \xi^{3}\right)+\left(K_{1}-K_{0}\right) /(1-\xi)^{3}>0$, thus the change of distorted region size $d \xi / d x$ or the level of distortion propagation of a ten-stage compressor $\xi(10)-\xi(0)$ would depend on the airfoil forces in both the distorted and undistorted regions. Therefore, it is vital to apply the appropriate/associated airfoil characteristics in the prediction of inlet distortion propagation, and the calculated results would be more realistic.

\section{CONCLUDING REMARKS}

The original integral method for investigating a compressor inlet distortion has been developed further, and utilizing the more appropriate and practical airfoil characteristics, we derive a novel method. The simulation indicates that results calculated using the previous integral method underestimated the level of distortion propagation as compared to 
the current novel integral method. The investigation of inlet parameters is also proceeded and the results suggest that the inlet distortion level and the inlet incidence angle have noticeable effects upon the downstream flow features, especially in the level of distortion propagation.

The present effort suggests that this simple method may be flourishing in the problems of strongly distorted flow and propagating stall in axial compressor. It is believed that using a more realistic and flexible velocity and pressure profiles could develop this approach further.

\section{ACKNOWLEDGMENTS}

The authors would like to thank HQ RSAF for permission to publish this work, their financial support, and encouragement. The first author wants to acknowledge Professor Frank Marble of California Institute of Technology for bringing the problem to the author's attention and for his helpful discussion.

\section{REFERENCES}

[1] J. P. Chen and W. R. Briley, "A parallel flow solver for unsteady multiple blade row turbomachinery simulations," in ASME Turbo Expo, New Orleans, La, USA, June 2001, ASME Paper 2001-GT-0348.

[2] N. A. Cumpsty and E. M. Greitzer, "A simple model for compressor stall cell propagation," ASME Journal of Engineering for Power, vol. 104, no. 2, pp. 170-176, 1982.

[3] H. W. Emmons, C. E. Pearson, and H. P. Grant, "Compressor surge and stall propagation," Transactions of the ASME, vol. 79, pp. 455-469, May 1955.

[4] E. M. Greitzer, "Review: axial compressor stall phenomena," ASME Journal of Fluids and Engineering, vol. 102, pp. 134151, June 1980.

[5] S. Jonnavithula, S. Thangam, and F. Sisto, "Computational and experimental study of stall propagation in axial compressors," AIAA Journal, vol. 28, no. 11, pp. 1945-1952, 1990.

[6] J. H. Kim, F. E. Marble, and C.-J. Kim, "Distorted inlet flow propagation in axial compressors," in Proc. 6th International Symposium on Transport Phenomena and Dynamics of Rotating Machinery, vol. 2, pp. 123-130, Honolulu, Hawaii, USA, February 1996.

[7] N. Y. Liu, E. Y.-K. Ng, H. N. Lim, and T. L. Tan, "Stall prediction of in-flight compressor due to flamming of refueling leakage near inlet," Journal of Computational Mechanics, vol. 30, no. 5-6, pp. 479-486, 2003.

[8] J. P. Longley and T. P. Hynes, "Stability of flow through multistage axial compressors," ASME Journal of Turbomachinery, vol. 112, pp. 126-132, January 1990.

[9] F. E. Marble, "Propagation of stall in a compressor blade row," Journal of the Aeronautical Sciences, vol. 22, no. 8, pp. 541-554, 1955.

[10] R. S. Mazzawy, "Multiple segment parallel compressor model for circumferential flow distortion," ASME Journal of Engineering for Power, vol. 99, no. 4, pp. 288-296, 1977.

[11] A. A. Mikolajczak and A. M. Pfeffer, Method to increase engine stability and tolerance to distortion, 1974, AGARD 72.

[12] E. Y.-K. Ng, N. Y. Liu, H. N. Lim, and T. L. Tan, "Study on the distorted inlet flow propagation in axial compressor using an integral method," Journal of Computational Mechanics, vol. 30, no. 1, pp. 1-11, 2002.
[13] G. A. Plourde and A. H. Stenning, "Attenuation of circumferential inlet distortion in multistage axial compressors," in Proc. AIAA 3rd Propulsion Joint Specialist Conference, Washington, DC, USA, July 1967, AIAA Paper 67-GT-415.

[14] C. Reid, "The response of axial flow compressors to intake flow distortion," in Proc. International Gas Turbine and Aeroengine Congress and Exhibition, New York, NY, USA, March 1969, ASME Paper 69-GT-29.

[15] G. K. Serovy and R. P. Dring, "Experimental test cases for turbines," in Test Cases for Computation of Internal Flows in Aero Engine Components, L. Fottner, Ed., pp. 322-330, Advisory Group for Aerospace Research and Development, Brussels, Belgium, July 1990, AGARD-AR-275.

[16] A. H. Stenning, "Inlet distortion effects in axial compressors," ASME Journal of Fluids Engineering, vol. 102, no. 3, pp. 7-13, 1980.

[17] I. H. Abbott and A. E. Von Doenhoff, Theory of Wing Sections Including a Summary of Airfoil Data, Dover, New York, NY, USA, 1959.

[18] S. R. Wellborn and R. A. Delaney, "Redesign of a 12-stage axial-flow compressor using multistage CFD," in ASME Turbo Expo, New Orleans, La, USA, June 2001, ASME Paper 2001GT-0315. 

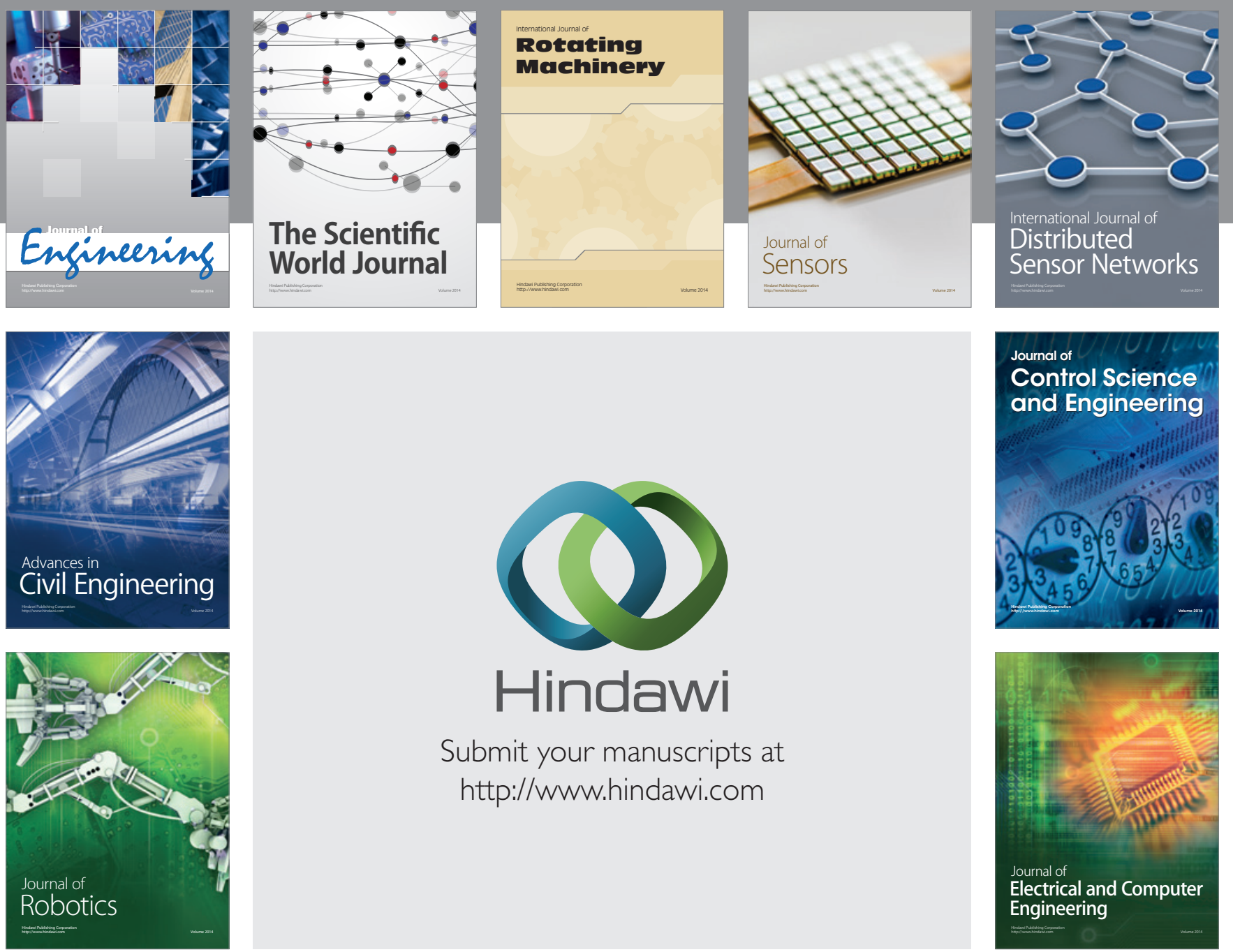

Submit your manuscripts at

http://www.hindawi.com
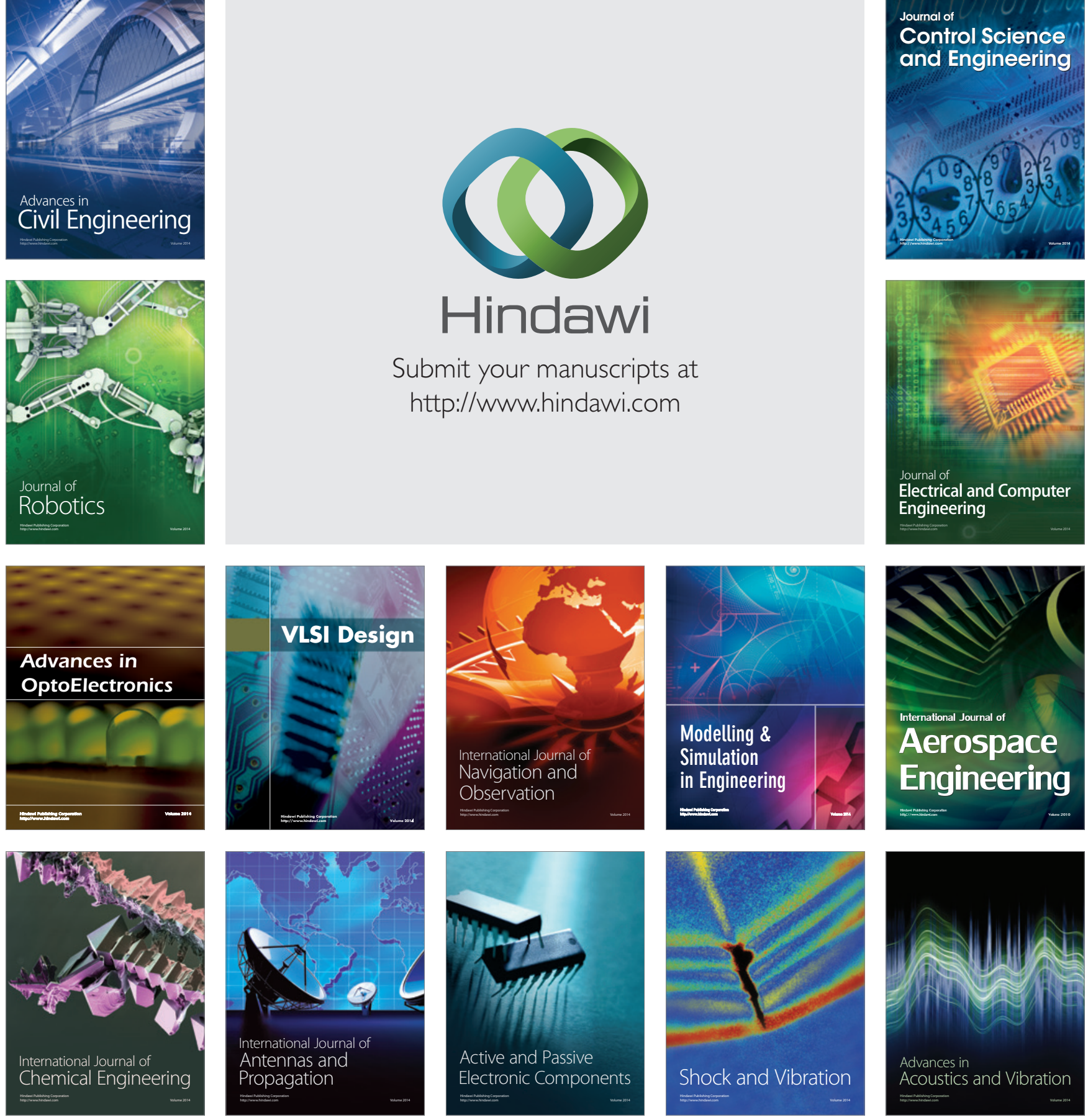